$05,08,12$

\title{
Эволюция триплетных парамагнитных центров в алмазах, получаемых спеканием детонационных наноалмазов при высоком давлении и температуре
}

\author{
() В.Ю. Осипов ${ }^{1}$, А.И. Шамес ${ }^{2}$, Н.Н. Ефимов ${ }^{3}$, Ф.М. Шахов ${ }^{1}$, С.В. Кидалов ${ }^{1}$, В.В. Минин ${ }^{3}$, А.Я. Вуль $^{1}$ \\ ${ }^{1}$ Физико-технический институт им. А.Ф. Иофрфе РАН, \\ Санкт-Петербург, Россия \\ ${ }^{2}$ Department of Physics, Ben-Gurion University of the Negev, \\ Be'er-Sheva, Israel \\ ${ }^{3}$ Институт общей и неорганической химии им. Н.С. Курнакова РАН, \\ Москва, Россия \\ E-mail: osipov@mail.ioffe.ru
}

(Поступила в Редакцию 11 сентября 2017 г.)

\begin{abstract}
Исследованы спектры электронного парамагнитного резонанса (ЭПР) триплетных центров в наноалмазах детонационного синтеза и синтезируемых из них при высоких давлениях и температурах алмазных монокристаллах субмикронных размеров. В спектрах ЭПР детонационных наноалмазов наблюдаются сигналы от отрицательно заряженных азот-вакансионных центров $(\mathrm{NV})^{-}$с $g$-фактором $g_{1}=4.24$ и мультивакансий с $g_{2}=4.00$. В спектрах алмазных микрокристаллов исчезают сигналы от $(\mathrm{NV})^{-}$центров, и на месте сигнала от мультивакансий обнаруживается квинтетный сигнал с $g=4.00$. Анализ формы и положения линии квинтета показал, что этот ЭПР сигнал обусловлен парами азотных центров замещения в алмазе, удаленных друг от друга на расстояния, не превышающие 0.7 нанометров, между которыми осуществляется сильное обменное взаимодействие. Сопоставление экспериментальных данных и результатов моделирования позволило определить параметры спин-гамильтониана обменно-связанных пар парамагнитных примесных атомов азота.
\end{abstract}

DOI: 10.21883 FTT.2018.04.45682.262

\section{1. Введение}

Одной из наиболее распространенных примесей в синтетических кристаллах алмаза является азот, замещающий атомы углерода в кристаллической решетке алмаза. При традиционном синтезе алмаза из графита в присутствии металла-катализатора при высоких давлениях и температурах (НРНТ синтез) источником азота является воздух $[1,2]$.

Атомы азота могут находиться в решетке алмаза в виде изолированных парамагнитных примесей, ${ }^{1}$ непарамагнитных положительно или отрицательно заряженных состояниях, непарамагнитных А центров, состоящих из двух атомов азота, расположенных в ближайших узлах решетки, азот-вакансионных центров, как нейтральных, так и отрицательно заряженных $(\mathrm{NV})^{-}$, и комплексов, состоящих из нескольких вакансий и атомов азота [3-5]. К последним относятся и мультивакансии, т. е. кластеры, состоящие из двух и более вакансий, организованные в линейные структуры с регибридизованными внутренними электронными орбиталями [4].

Характерные ЭПР сигналы регистрируются для „запрещенных“ $\left(\Delta M_{s}=2\right)^{2}$ переходов между зееманов-

\footnotetext{
1 Здесь подразумевается замещающий атом $\mathrm{N}_{s}$ в нейтральном состоянии, обозначаемый как С или Р1 центр в классификаторе типов дефектов в алмазе.

2 Здесь $\Delta M_{s}$ - изменение проекции магнитного момента на направление магнитного поля, т.е. магнитного квантового числа спина, при поглощении кванта излучения.
}

скими уровнями парамагнитных центров в триплетных спиновых состояниях $(S=1)$, которыми являются $\mathrm{NV}^{-}$-центры, мультивакансии и пары обменно связанных Р1 центров. Для первых двух типов центров спектры ЭПР хорошо известны [4]. О наблюдении необычной сверхтонкой структуры спектров ЭПР центров, состоящих из двух атомов азота, сообщалось только в работах $[6,7]$. Спектры представляли собой набор из пяти эквидистантно расположенных линий в области магнитных полей $1 / 2 H_{\text {res }},{ }^{3}$ соответствующих $g$-фактору $g=4$ (так называемые половинные магнитные поля, ПП).

ЭПР сигналы, характерные для „запрещенных“ $\Delta M_{s}=2$ переходов в триплетных центрах, были также обнаружены в получаемых по взрывной технологии детонационных наноалмазах (ДНА) со средним размером частиц 4-5 nm $[8,9]$ и в наночастицах синтетических флуоресцентных алмазов, получаемых механическим размельчением крупных кристаллов, предварительно облученных электронным пучком [10].

Синтез НРНТ алмазных кристаллов связан с использованием металлических катализаторов, растворяющих в себе графит. При выращивании НРНТ алмазов в качестве катализаторов чаще всего используются металлы группы железа и их сплавы. Алмазы, выращенные таким способом, содержат большое количество пара- и

\footnotetext{
${ }^{3}$ В диапазоне микроволновых частот 9.4-9.9 GHz резонансное магнитное поле $H_{\text {res }}$, соответствующее основному сигналу ЭПР парамагнитных центров со спином $S=1 / 2$, лежит в интервале $318-335 \mathrm{mT}$.
} 
ферромагнитных примесей [11] и непригодны для ряда применений.

Недавно в работах разных групп исследователей была продемонстрирована возможность образования монокристаллов алмаза субмикронных и микронных размеров из ДНА при высоких давлениях и температурах при использовании в качестве реакционной смеси порошка ДНА вместе с предельным ациклическим углеводородом, одноосновными или многоосновными спиртами [12,13], или нафталином [14]. Использование этой технологии дает возможность получать алмазные микрокристаллиты повышенной чистоты с малой зольностью $(<0.1 \mathrm{wt} \%)$, определяемой лишь химической чистотой исходного ДНА.

ЭПР спектры таких алмазов свободны, как правило, от интенсивных сигналов в магнитных полях ниже $H_{r e s}$, ассоциируемых с пара- и ферромагнитными примесями. При комнатной температуре в ЭПР спектрах исследуемых образцов в области ПП были обнаружены новые сигналы ЭПР, характеризуемые $g$-фактором $g=4.00$ и хорошо разрешенной сверхтонкой структурой из 5 линий. Последние существенно отличаются от ранее наблюдавшихся в ДНА и наноразмерных НРНТ алмазах ЭПР сигналов в том же диапазоне магнитных полей, связываемых с NV центрами и мультивакансиями $[9,10]$.

В данной работе мы приводим результаты исследования ЭПР, которые отражают эволюцию триплетных центров при формировании субмикронных монокристаллов алмаза из наночастиц ДНА.

\section{2. Образцы и методика эксперимента}

Порошкообразный образец, представляющий из себя набор микрокристаллических алмазов разных размеров и степеней агрегирования, был получен путем спекания порошка детонационных наноалмазов при высоком давлении $(P \approx 7 \mathrm{GPa})$ и температуре $T \geq 1350^{\circ} \mathrm{C}$ с добавлением в реакционную смесь этилового спирта, аналогично методу, описанному в работах $[12,13]$. Схема снаряженного контейнера в камере высокого давления в сборе представлена в работе [15]. Весовая доля этанола в контейнере не превышала $35 \mathrm{wt} \%$ от массы ДНА. Нагрев осуществлялся после приложения давления. Продолжительность синтеза составляла $\sim 10 \mathrm{~s}$. Скорость нагрева составляла $\sim 200^{\circ} \mathrm{C} / \mathrm{s}$. Герметичность камеры обеспечивала избыточное давление продуктов разложения этанола и поверхностных функциональных групп, десорбирующихся с поверхности ДНА, внутри контейнера. Синтезированный алмазный порошок отделялся от остатков материала контейнера промывкой в $\mathrm{HBr}$ с последующим разделением по плотности графита, являющегося материалом нагревателя, и алмазов в бромоформе $\mathrm{CHBr}_{3}$ (тяжелой жидкости с $\rho=2.9 \mathrm{~g} / \mathrm{cm}^{3}$ ). Практически весь (до $\sim 90-95 \%$ ) засыпаемый в контейнер исходный порошок ДНА трансформировался в алмазные кристаллы субмикронных размеров. Детальные исследования были проведены на образце, полученном

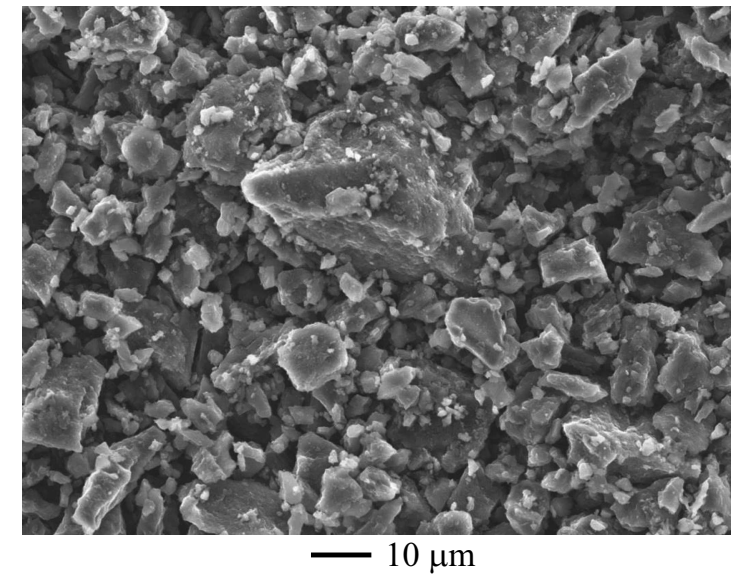

Рис. 1. Изображение микронных и субмикронных алмазных частиц, полученное с помощью сканирующей электронной микроскопии. Ускоряющее напряжение $15 \mathrm{kV}$. Длина масштабной полоски $10 \mu \mathrm{m}$.

при температуре спекания $1380^{\circ} \mathrm{C}(D 1)$. Кубическую алмазную решетку полученных монокристаллов идентифицировали методом рентгеновской дифракции с размером области когерентного рассеяния для крупных кристаллов $\sim 80-90 \mathrm{~nm}$. По данным динамического рассеяния света, размер алмазных частиц в исследованном образце составляет $40-200 \mathrm{~nm}$, хотя на изображениях, полученных методом сканирующей электронной микроскопии, видны и бесформенные агрегаты кристаллов размером до $10 \mu \mathrm{m}$ (рис. 1).

Спектры ЭПР исходного ДНА и синтезированных микрокристаллов регистрировали при комнатной температуре $(К Т, T \approx 295 \mathrm{~K})$ в $X$-диапазоне $(9.8 \mathrm{GHz})$ с помощью ЭПР спектрометра Bruker ELEXSYS E-680X. Порошки ДНА и микроалмазов в количествах $10-15 \mathrm{mg}$ засыпали в кварцевую трубку (Wilmad ${ }^{\circledR}$ ) с внешним диаметром $4 \mathrm{~mm}$. Спектры основных интенсивных сигналов $(g=2.00)$ и значительно более слабых сигналов в области ПП $(g=4.00)$ записывали при мощности микроволнового излучения в диапазоне $0.001-36 \mathrm{~mW}$, амплитуде модуляции магнитного поля $0.01-1 \mathrm{mT}$, частоте модуляции $100 \mathrm{kHz}$, количестве когерентных накоплений сигнала 1-200, которое для сигналов малой интенсивности определялось достижением приемлемого соотношения сигнал/шум в записываемом спектре. Сигналы малой интенсивности с $g$-фактором $g \approx 4$ регистрировали при мощности $32 \mathrm{~mW}$ и модуляции $1 \mathrm{mT}$. Обработку спектров ЭПР и симуляцию спектров поликристаллов в области ПП производили с использованием программ Win-EPR (Bruker) и EasySpin [16] соответственно.

\section{3. Результаты эксперимента и их обсуждение}

Основные сигналы ЭПР синтезированных субмикрокристаллических алмазов соответствуют сигналу 
P1 центров. Этот сигнал состоит из центрального с $g$-фактором $g=2.0024$ и двух симметрично-расположенных сателлитов [17]. Такие сигналы характерны для спектров ЭПР порошкообразных микрокристаллических образцов, содержащих парамагнитные центры с электронным спином неспаренной антисвязывающей орбитали $S=1 / 2$, который взаимодействует с ядерным спином $I=1\left({ }^{14} \mathrm{~N}\right)$ атома азота. Эволюция основных сигналов ЭПР при трансформации ДНА в субмикронные и микронные алмазные частицы - от интенсивного квази-лоренцевого синглета в ДНА до менее интенсивных характерных сигналов Р1 центров в микронных алмазных частицах - детально описана в работе [17].

Не меньший интерес представляют и изменения, произошедшие в спектрах ЭПР в области ПП в результате синтеза из ДНА алмазных кристаллитов микронного размера. Характерной особенностью ЭПР спектров ДНА в $X$-диапазоне в области ПП является дублет, состоящий из частотно зависимого сигнала с $g=4.24-4.28$ и сигнала с $g=4.00$, практически не зависящего от частоты. Первый сигнал связывают с так называемыми „запрещенными“ переходами $\Delta M_{S}=2$ в ЭПР спектре порошкообразного образца, содержащего триплетные $\mathrm{NV}^{-}$центры. Второй сигнал ПП дублета в ДНА относится к $\Delta M_{S}=2$ переходам в спектрах ЭПР мультивакансий, образованных парой оборванных $s p^{3}$ связей, взаимодействующих по дипольному или обменному механизмам [10,18]. Упомянутый дублет отчетливо наблюдается в ЭПР спектре порошка нанометровых кристаллитов ДНА, из которых был синтезирован исследуемый образец $D 1$ (сплошная кривая, рис. 2). Однако в ЭПР спектре порошкообразного образца $D 1$ (после НРНТ синтеза) сигнал с $g=4.24$ практически исчезает, а на месте сигнала с $g=4.00$ от мультивакансий возникает новый сигнал, представляющий из себя квинтет квази-эквидистантных линий с расщеплениями порядка $1.6 \mathrm{mT}$ (штриховая кривая, рис. 2). Интегральные интенсивности линий, соответствующих сигналам в области $g=4.24$ и $g=4.00$ в спектрах образцов ДНА и $D 1$ в 500 и 8500 раз меньше интенсивности основной линии от парамагнитных центров с $S=1 / 2$ в образце ДНА.

Природа подобных сигналов ЭПР, наблюдаемых в спектрах синтетических алмазов, полученных НРНТ методом из графита, содержащих высокие концентрации примесного азота, была детально исследована при низких температурах в работе [7]. Было установлено, что источником сверхтонкого квинтета в половинном поле являются центры, обозначаемые как NOC4, в которых два центра Р1 оказываются связанными взаимодействием, имеющим в основном диполь-дипольную природу (для центров, расстояния между которыми превышают $0.7 \mathrm{~nm}$ ). Для более близко расположенных центров взаимодействие может носить смешанный диполь-дипольный и обменный характер. Поскольку резонансное поле центральной линии квинтета в области ПП в ЭПР

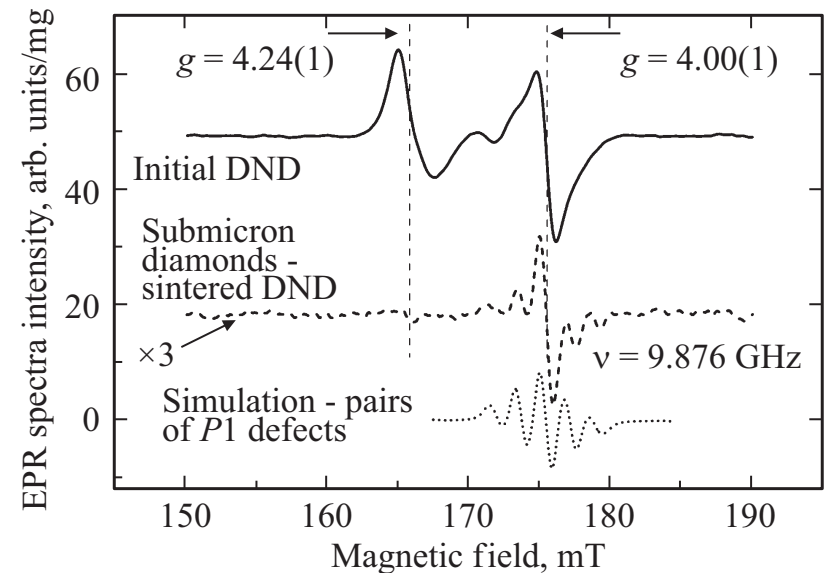

Рис. 2. Спектры ЭПР в ПП (область $g=4.00$ ), зарегистрированные при КТ и микроволновой частоте $v=9.876 \mathrm{GHz}$. Сверху вниз: сплошная кривая - порошок исходного ДНА, штриховая кривая - порошок $D 1$, пунктирная кривая - рассчитанный спектр ЭПР хаотически ориентированных пар взаимодействующих P1 центров, полученный при помощи программы EasySpin c параметрами спин-гамильтониана (1) $g_{\text {iso }}=2.0024, A_{1 x}=A_{2 x}=A_{1 y}=A_{2 y}$ $=2.9 \mathrm{mT} ; A_{1 z}=A_{2 z}=4.1 \mathrm{mT}, D=150 \cdot 10^{-4} \mathrm{~cm}^{-1}, E=0$, ширина индивидуальной линии лоренцевского типа $H_{p p}=1 \mathrm{mT}$. Вертикальные прерывистые линии указывают положения сигналов с $g=4.24$ и 4.00. Спектры, показанные сплошной и штриховой линиями, зарегистрированы в одинаковых экспериментальных условиях. Интенсивности нормированы на единицу веса, широкие фоновые линии вычтены. Интенсивность рассчитанного спектра (нижняя пунктирная кривая) нормирована на интенсивность крайней низкополевой линии (при $~ 172 \mathrm{mT}$ ) экспериментального спектра образца $D 1$.

спектрах образцов было найдено изотропным [7], авторы предположили, что анизотропный вклад в спингамильтониан (CГ) центров NOC4 отсутствует, а смешивание зеемановских триплетных подуровней с $M_{S}=0$ и $M_{S}= \pm 1$ происходит только за счет сверхтонкого взаимодействия. В этом случае интенсивности наблюдаемых „запрещенных“ сигналов пропорциональны квадрату отношения константы сверхтонкого взаимодействия $A$ к радиочастотному кванту $h v$, т.е. являются величинами $\sim 10^{-4}$ от интенсивности соответствующих ,разрешенных“ сигналов.

Подобный СГ исчерпывающим образом объясняет все наблюдаемые особенности в спектрах ЭПР, отмеченные в работе [7]. Однако применение этой модели к описанию наблюдаемого нами ЭПР спектра ПП образца не соответствует экспериментальным фактам. Так, в работе [7] квинтетный сигнал в ПП регистрировался только при температурах, не превышающих $40 \mathrm{~K}$, что свидетельствует о его малой интенсивности. В противоположность этому, в нашем случае ПП квинтет надежно наблюдается при комнатной температуре, т. е. его интенсивность, как минимум, на порядок выше интенсивности сигналов ПП в образцах, описанных в работе [7]. Ис- 
ключая предположение о сверхвысоких концентрациях взаимодействующих пар Р1, можно предположить, что в нашем случае смешивание зеемановских подуровней осуществляется не столько сверхтонким взаимодействием, сколько анизотропным взаимодействием типа SDS с $D \neq 0$. В этом случае СГ триплетного центра, образованного парой взаимодействующих Р1 центров описывается уравнением

$$
\begin{aligned}
\hat{\mathbf{H}}= & g \mu_{\mathrm{B}} \mathbf{B S}+D\left[S_{Z}^{2}-\frac{1}{3} S(S+1)\right]+E\left(S_{X}^{2}+S_{Y}^{2}\right) \\
& +\mathbf{S}\left(\mathbf{A}_{1} \mathbf{I}_{1}+\mathbf{A}_{2} \mathbf{I}_{2}\right),
\end{aligned}
$$

где электронный спин $S=1$, ядерные спины ${ }^{14} \mathrm{~N}$ $I_{1}=I_{2}=1$, первый член описывает зеемановское взаимодействие спина с внешним магнитным полем, $D$ и $E$ - параметры расщепления триплетного уровня в нулевом магнитном поле, а четвертый член описывает анизотропное сверхтонкое взаимодействие ядерных спинов Р1 центров с электронным спином $S$.

Пунктирная кривая на рис. 2 демонстрирует ЭПР спектр в области $g=4.00$, рассчитанный для пар взаимодействующих P1 центров с использованием программы EasySpin [16] при следующих параметpax СГ: $g_{\text {iso }}=2.0024, A_{1 x}=A_{2 x}=A_{1 y}=A_{2 y}=2.9 \mathrm{mT}$. $A_{1 z}=A_{2 z}=4.1 \mathrm{mT}, \quad D=150 \cdot 10^{-4} \mathrm{~cm}^{-1}, \quad E=0$, ширина индивидуальной линии лоренцевского типа $\Delta H_{p p}=1 \mathrm{mT}, v=9.876 \mathrm{GHz}$. Сравнение штриховой и пунктирной кривых на рис. 2 свидетельствует о том, что избранная модель и формула (1) хорошо описывают наблюдаемый квинтетный ЭПР сигнал.

Основным отличием экспериментального и рассчитанного сигналов на рис. 2 является аномально большая интенсивность центральной компоненты (см. ниже). Поскольку в экспериментальном ЭПР спектре не были обнаружены сигналы, соответствующие „разрешенным“ $\Delta M_{S}=1$ переходам от пар взаимодействующих Р1 центров, ${ }^{4}$ непосредственная оценка параметров нулевого расщепления в СГ (1) (определяемого из расстояний между высоко- и низкополевыми компонентами порошкообразного спектра „разрешенных“ сигналов, либо из отношения интегральных интенсивностей „запрещенных“ и „разрешенных“ сигналов) не представляется возможной. Тем не менее оценить значения этих параметров можно посредством сравнения экспериментального спектра с набором расчетных спектров ПП, полученных при варьировании параметров нулевого расщепления.

На рис. 3, $a$, показана серия спектров, рассчитанных с приведенными выше фиксированными параметрами СГ, за исключением параметра нулевого расщепления $D$, который меняется от $D=23 \cdot 10^{-4} \mathrm{~cm}^{-1}$ (нижняя кри-

\footnotetext{
${ }^{4}$ Как правило, в спектрах ЭПР порошков микрокристаллического алмаза сигналы, соответствующие „разрешенным“ переходам триплетных пар Р1 (со спином $S=1$ ), являются трудно наблюдаемыми, так как они ориентационно уширены и расположены вблизи значительно более интенсивных сигналов от преобладающих одиночных P1 центров в районе $g=2.00$.
}

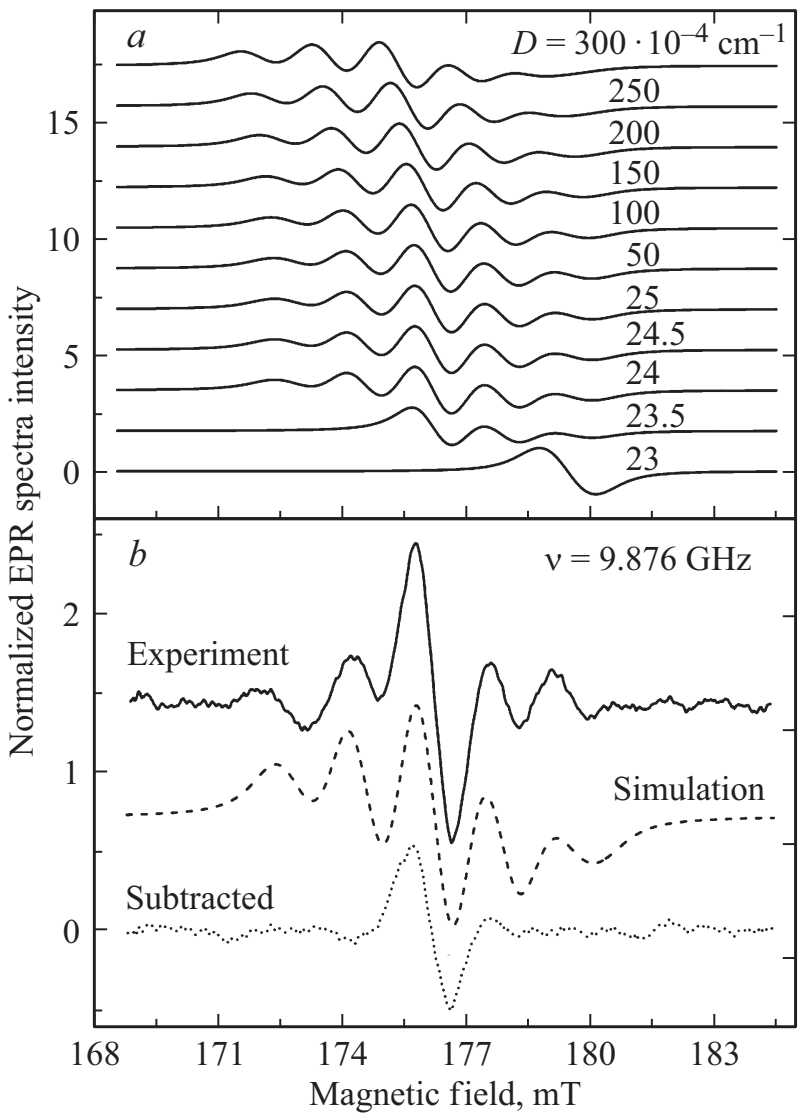

Рис. 3. $a-$ Спектры ЭПР порошков субмикрокристаллических алмазов, содержащих пары взаимодействующих Р1 центров, рассчитанные программой EasySpin с параметрами спин-гамильтониана (1) $g_{\text {iso }}=2.0024, A_{1 x}=A_{2 x}=A_{1 y}=A_{2 y}$ $=2.9 \mathrm{mT}, A_{1 z}=A_{2 z}=4.1 \mathrm{mT}, E=0$, ширина индивидуальной линии лоренцевского типа $\Delta H_{p p}=1 \mathrm{mT}$, при частоте $v=9.876 \mathrm{GHz}$ и разных значениях параметра $D$. Величина параметра $D$ увеличивается от нижнего спектра $\left(D=23 \cdot 10^{-4} \mathrm{~cm}^{-1}\right)$ к верхнему $\left(D=300 \cdot 10^{-4} \mathrm{~cm}^{-1}\right)$, значения, соответствующие каждому спектру, указаны в правой части рисунка. $b-$ спектры ЭПР, сверху вниз: сплошная кривая - экспериментальный спектр ПП исследуемого порошка $D 1$, зарегистрированный при КТ и микроволновой частоте $v=9.876 \mathrm{GHz}$; штриховая кривая - рассчитанный спектр субмикрокристаллического порошка для пары взаимодействующих P1 центров с параметром $D=150 \cdot 10^{-4} \mathrm{~cm}^{-1}$, интенсивность рассчитанного спектра (штриховая кривая) нормирована на интенсивность крайней низкополевой линии экспериментального спектра образца $D 1$; пунктирная кривая спектр, полученный вычитанием рассчитанного спектра из экспериментального.

вая) до $D=300 \cdot 10^{-4} \mathrm{~cm}^{-1}$ (верхняя кривая). Из этого рисунка хорошо видно, что спектр в ПП, подобный экспериментальному спектру, соответствует значениям $D$, лежащим в диапазоне от $D=24 \cdot 10^{-4} \mathrm{~cm}^{-1}$ (при котором начинает наблюдаться квинтетная структура спектра) до $D=150 \cdot 10^{-4} \mathrm{~cm}^{-1}$. При значениях $D>150 \cdot 10^{-4} \mathrm{~cm}^{-1}$ центральная линия квинтета начинает сдвигаться в направлении бо́льших $g$-факторов, 
отличающихся от экспериментально определенного $g$-фактора в ЭПР спектре на величину, существенно превышающую установленную экспериментальную погрешность.

Ответ на вопрос о выборе величины нулевого расщепления $D$ из определенного выше диапазона значений не может быть получен только на основании полученных из рассчитанных кривых данных о форме и $g$-факторах спектров в ПП. Тем не менее сам факт наблюдения квинтетных сигналов при комнатной температуре указывает на правильность оценки величины нулевого расщепления по ее верхнему пределу, т.е. $D \approx 150 \cdot 10^{-4} \mathrm{~cm}^{-1}$. Эта величина, по данным работ $[6,7]$, свидетельствует в пользу правильности выбора СГ, описываемого формулой (1), и позволяет заключить, что расстояние между индивидуальными спинами в таком триплетном центре не превышает $0.7 \mathrm{~nm} .^{5}$ Полученное значение параметра $D$ в сочетании с вышеупомянутыми данными об интегральных интенсивностях сигналов в ПП позволяют оценить концентрацию центров, ответственных за квинтет в спектрах образца $D 1$. Используя образец флуоресцентного синтетического микрокристаллического алмаза с известным содержанием $\mathrm{NV}^{-}$центров в качестве стандарта [18], было установлено, что концентрация $\mathrm{NV}^{-}$центров в исходном образце ДНА не превышает $2.4 \mathrm{ppm}$. Поскольку интенсивности ,запрещенных“ переходов пропорциональны $(D / h v)^{2}$, менее интенсивному квинтетному сигналу в образце $D 1$ соответствует концентрация триплетных центров 5.6 ppm.

Пунктирная кривая на рис. $3, b$ представляет собой результат вычитания спектра, рассчитанного при значении $D=150 \cdot 10^{-4} \mathrm{~cm}^{-1}$ (штриховая кривая), из экспериментального спектра образца $D 1$ (сплошная кривая). Подгоночный масштабный коэффициент при вычитании был подобран таким образом, чтобы минимизировать разностные сигналы от боковых сверхтонких линий квинтета. Опираясь на этот результат, можно с уверенностью предположить, что аномальная интенсивность центральной линии квинтета обусловлена суперпозицией двух сигналов: сигнала от взаимодействующих пар P1 и остаточного синглетного сигнала с тем же (в пределах экспериментальной ошибки) $g$-фактором $g=4.00$, наблюдаемого в спектрах исходного ДНА.

Суммируя все полученные данные можно заключить, что в процессе описываемого синтеза субмикронных алмазов триплетные центры, имеющиеся в исходных ДНА, претерпевают следующие изменения: содержание триплетных $\mathrm{NV}^{-}$центров при спекании падает ниже $30 \mathrm{ppb}$ - нижнего предела обнаружения; содержание триплетных мультивакансий резко падает; появляются новые триплетные центры типа NOC4, представляющие собой взаимодействующие пары P1 центров, находящихся, в отличие от описанных в работе [7] центров NOC4, на расстояниях не более $0.7 \mathrm{~nm}$.

\footnotetext{
${ }^{5}$ Как правило, для простых триплетных центров $S=1$ параметр $D$ в спин-гамильтониане обратно пропорционален третьей степени расстояния между индивидуальными спинами 1/2 в центре [4].
}

\section{4. Заключительные замечания и выводы}

Обнаруженные изменения в типе и количестве триплетных центров в синтезируемых микрокристаллах алмазов (по сравнению с наблюдаемым в исходном порошке ДНА) происходят параллельно с падением концентрации оборванных связей и других типов парамагнитных дефектов со спином $S=1 / 2$.

В работе [17] было показано, что концентрация всех типов дефектов со спином $S=1 / 2$ падает от $6.3 \cdot 10^{19} \mathrm{~g}^{-1}(\sim 1250 \mathrm{ppm})$ до $8.2 \cdot 10^{18} \mathrm{~g}^{-1}$ $(\sim 165 \mathrm{ppm})$ при спекании ДНА в кристаллиты размером 40-200 nm. Из них как минимум $35-40 \%$ связано с парамагнитным азотом (Р1 центры) [17]. Остальные парамагнитные центры с $S=1 / 2$ обусловлены дефектами неазотного происхождения, в частности отрицательнозаряженными вакансиями. Дефектность образующихся монокристаллов размером до $200 \mathrm{~nm}$ оказывается ниже, чем у ДНА, за счет агрегации части Р1 центров в непарамагнитные кластеры. Наблюдение сигналов от пар близких Р1 центров со спином $S=1$ становится возможным на фоне общего снижения интенсивности ЭПР сигналов от парамагнитных триплетных центров другого происхождения, прежде всего, мультивакансий.

На рис. 4. схематически показана трансформация всех типов парамагнитных дефектов со спинами как $S=1$, так и $S=1 / 2$ при спекании ДНА, приводящая к появлению азотных пар триплетного типа со спином $S=1$. Использование высоких давлений и температур для обесцвечивания и уменьшения дефектности монокристаллов алмаза ювелирного качества, вообще говоря, известно. Однако агрегация дефектов в кластеры при высоких давлениях и температурах $\sim 1400^{\circ} \mathrm{C}$, как правило, происходит за длительное время (от получаса и выше). В процессе описанного выше синтеза падение концентрации основных дефектов со спином $S=1 / 2$ и существенная эволюция всех типов триплетных центров осуществляются за время $\leq 10 \mathrm{~s}$. Столь быстрые изменения нельзя объяснить одной только диффузией атомов азота и вакансий в решетке алмаза при давлении $7 \mathrm{GPa}$ и температурах $\sim 1400^{\circ} \mathrm{C}^{6}$ Ясно, что для объяснения резких изменений в составе ансамбля всех триплетных центров в решетке следует привлекать потенциально более быстрые процессы в системе. К таким процессам можно отнести и химические реакции между углеродом ДНА и продуктами разложения этанола, и рекристаллизацию ядер ДНА в процессе синтеза крупных монокристаллов. Химические реакции между ДНА и активными реакционными агентами (например, карбидообразующими металлами) в условиях бо́льших

\footnotetext{
${ }^{6}$ В работе [17] нами отмечалось, что при НРНТ обработке ДНА в матрице из меди при давлении $7 \mathrm{GPa}$ и температуре $1400-1500^{\circ} \mathrm{C}$ существенного (более чем на 5-7\%) уменьшения концентрации основных парамагнитных центров в ДНА не происходит даже за 20-30s обработки, что говорит об отсутствии агрегации парамагнитного азота в непарамагнитные комплексы в кристаллитах ДНА в этих условиях.
} 


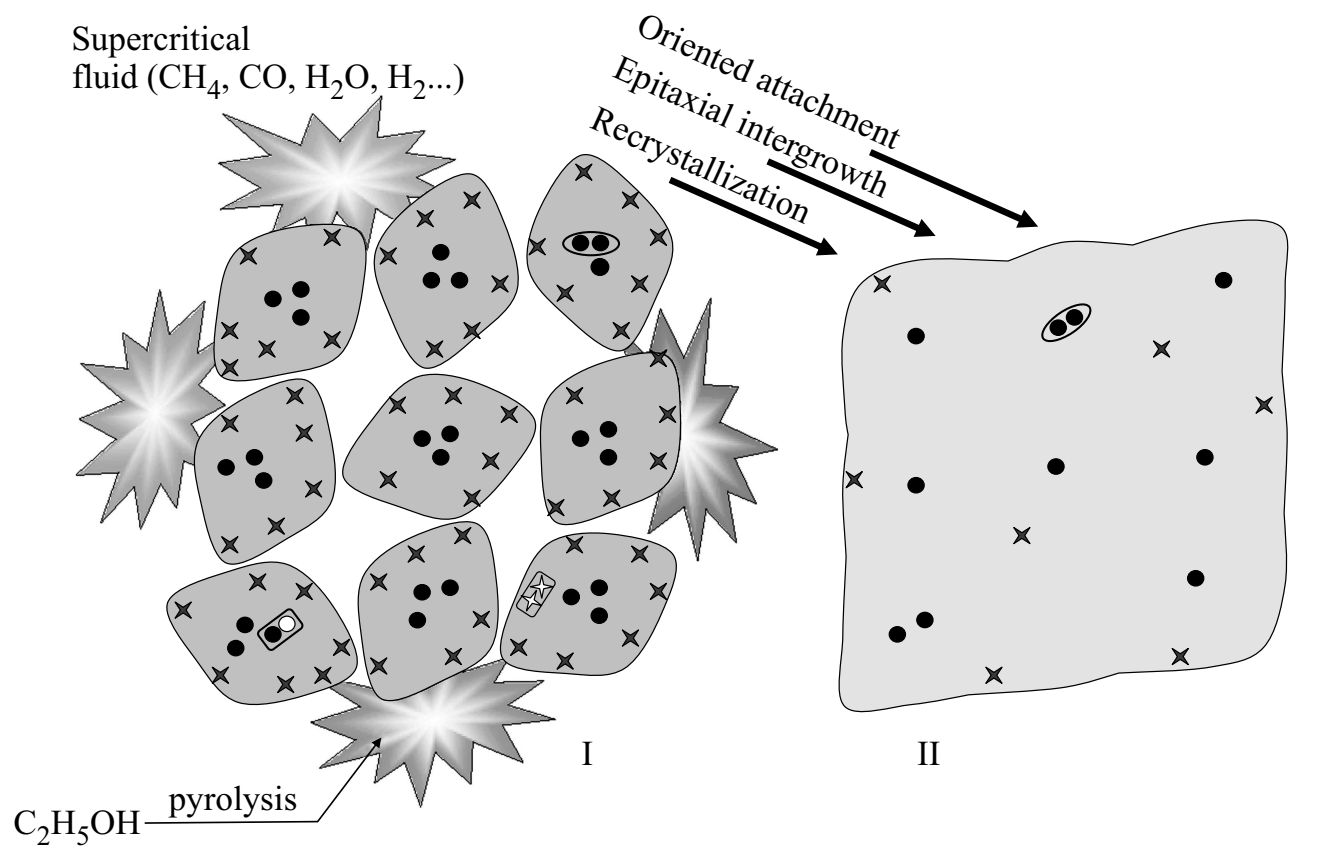

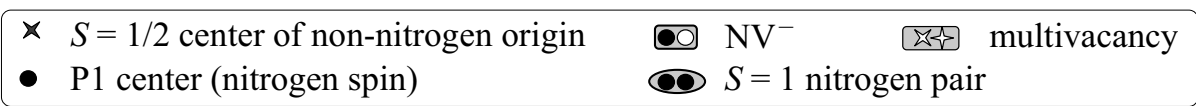

Рис. 4. Схема трансформации простых $S=1 / 2$ и „сложных“ $S=1$ центров $\left(\mathrm{NV}^{-}\right.$, мультивакансии, азотные пары) при образовании субмикронных кристаллов алмаза из частиц ДНА в результате спекания при высоком давлении и температуре. I - частицы ДНА до (и в процессе) спекания вместе со сверхкритическими флюидами состава С-H-O; II - субмикронный кристалл алмаза, образовавшийся в результате НРНТ спекания.

давлений и температур обычно проходят быстро, и за время $\sim 10-30$ s материал ДНА обычно полностью расходуется при избытке металлического компонента. В случае этанола активными реакционными агентами могут выступать простейшие структурные единицы и составные части этой молекулы, такие как $\mathrm{CH}_{4}, \mathrm{CO}$, $\mathrm{H}_{2} \mathrm{O}, \mathrm{H}_{2}$ и т. п, образующиеся в результате термического разложения (рис. 4).

Температура $\sim 1380^{\circ} \mathrm{C}$, при которой формируются алмазные кристаллы субмикронных размеров, существенно превышает температуру начала эффективного разложения используемых углеводородов, находящихся в состоянии сверхкритических флюидов [15]. Часть продуктов разложения, по всей видимости, находится в виде радикалов $\mathrm{CH}_{3}^{\bullet}$ - производных метана и, возможно, молекулярного водорода. При температурах $>800^{\circ} \mathrm{C}$ и давлении $\sim 7 \mathrm{GPa}$ этанол должен уже находиться в состоянии сверхкритического флюида, свободно протекающего в порошке ДНА [15]. Однако при температурах спекания вплоть до $\sim 1250-1300^{\circ} \mathrm{C}$ синтез микрокристаллических алмазов еще не начинается (или он происходит лишь частично в малом объеме) из-за отсутствия подходящих условий, способствующих эффективному росту алмаза. Водород при температурах $>1350^{\circ} \mathrm{C}$ играет роль буферного газа, содействующего росту новой алмазной фазы, а также может способствовать рекристаллизации поверхностного слоя алмазных ядер ДНА на глубину до $1-2 \mathrm{~nm}$. Как и в методе газофазного химического осаждения (CVD), наличие простейших углеводородных $s p^{3}$-радикалов $\mathrm{CH}_{3}^{\bullet}$ в буферной среде с избытком водорода способствует росту алмазной фазы в межчастичном пространстве (между частицами ДНА) и на самих нанометровых частицах ДНА, при этом обеспечивая ориентирование и взаимное сращивание частиц ДНА с одинаковой кристаллографической ориентацией. Кроме того, избыточное внутреннее давление (до 3-4 GPa [15]) газообразных компонент в сверхкритическом состоянии, по всей видимости, способствует вращению и повороту нанометровых кристаллов ДНА при их сращивании по механизму ориентированного присоединения [13]. В нашем исследовании, однако, происходит формирование алмазных монокристаллов субмикронных размеров из исходных частиц ДНА. При этом весовая доля углерода из этанола в образующихся кристаллах алмазов не превышает $12-15 \%$. Это свидетельствует о полном расходовании углерода этанола в ходе заращивания межчастичных промежутков и доращивания кристаллических интерфейсов в процессе спекания ДНА с одновременным образованием алмазных частиц субмикронных размеров (рис. 4). При этом основные изменения в типах парамагнитных триплетных центров, наблюдаемых в системе, происходят в алмазной 
решетке, формирующейся из нанокристаллитов ДНА. Исчезновение сигналов ЭПР с $g$-факторами $g_{1}=4.24$ и $g_{2}=4.00$ и появление на месте последнего квинтетного сигнала ЭПР от дефектов типа NOC4 может служить своеобразным маркером алмазов, полученных описанным выше способом в результате сращивания частиц ДНА.

Обнаруженная сверхтонкая структура сигнала триплетного центра типа NOC4 с уширенными индивидуальными линиями квинтета $\left(\Delta H_{p p} \sim 1.0 \mathrm{mT} v s\right.$. $\sim 0.3 \mathrm{mT}[7])$ свидетельствует, прежде всего, о малых (до $0.7 \mathrm{~nm}$ ) расстояниях между атомами азота замещения в парах, при которых становится существенным обменное взаимодействие. Уширение линий сверхтонкой структуры квинтета могло бы также происходить изза наличия „газа“ парамагнитных дефектов, окружающих эти пары, и действующего на них по механизму диполь-дипольного взаимодействия. Однако малая ширина центрального сигнала ЭПР от Р1 центров $\left(\Delta H_{p p}=0.23 \mathrm{mT}\right)$, в $\sim 4$ раза меньшая, чем ширина индивидуальных линий в квинтете, и малая концентрация парамагнитных дефектов в том же образце ( 165 ppm) [17], свидетельствуют в пользу первого предположения.

Работа выполнена при финансовой поддержке Российского научного фонда (проект 14-13-00795 „Синтез оптически активных материалов на основе наноалмазов, модифицированных ионами $3 d-4 f$ элементов“").

\section{Список литературы}

[1] A.M. Zaitsev. Optical Properties of Diamond: A Data Handbook. Springer-Verlag, Berlin-Heidelberg (2001). $503 \mathrm{p}$.

[2] I.A. Dobrinets, V.G. Vins, A.M. Zaitsev. HPHT-Treated Diamonds. Diamonds Forever. Springer Series in Materials Science 181. Springer-Verlag, Berlin-Heidelberg (2013). XIX, $257 \mathrm{p}$.

[3] W.V. Smith, P.P. Sorokin, I.L. Gelles, G.J. Lasher. Phys. Rev. 115, 1546 (1959).

[4] J.H.N. Loubser, J.A. van Wyk. Rep. Prog. Phys. 41, 1201 (1978).

[5] K.M. Etmimi, M.E. Ahmed, P.R. Briddon, J.P. Goss, A.M. Gsiea. Phys. Rev. B 79, 205207 (2009).

[6] V.A. Nadolinny, A.P. Yelisseyev, A.G. Badalyan, J.M. Baker, D.J. Twitchen, M.E. Newton, A. Hofstaetter, B. Feigelson. Diam. Relat. Mat. 8, 1565 (1999).

[7] V.A. Nadolinny, A.P. Yelisseyev, J.M. Baker, D.J. Twitchen, M.E. Newton, A. Hofstaetter, B. Feigelson. Phys. Rev. B 60, 5392 (1999).

[8] A.I. Shames, V.Yu. Osipov, H.J. von Bardeleben, A.Ya. Vul'. J. Phys.: Cond. Matter 24, 22, 225302 (2012).

[9] V.Yu. Osipov, A.I. Shames, T. Enoki, K. Takai, M.V. Baidakova, A.Ya. Vul'. Diam. Relat. Mat. 16, 12, 2035 (2007).

[10] A.I. Shames, V.Yu. Osipov, H.J. von Bardeleben, J.-P. Boudou, F. Treussart, A.Ya. Vul'. Appl. Phys. Lett. 104, 063107 (2014).
[11] F.M. Shakhov, A.M. Abyzov, S.V. Kidalov, A.A. Krasilin, E. Lähderanta, V.T. Lebedev, D.V. Shamshur, K. Takai. Phys. Chem. Solids 103, 224 (2017).

[12] С.В. Кидалов, Ф.М. Шахов, А.В. Швидченко, А.Н. Смирнов, В.В. Соколов, М.А. Яговкина, А.Я. Вуль. Письма в ЖТФ 43, 1, 21 (2017).

[13] A.T. Dideikin, E.D. Eidelman, S.V. Kidalov, D.A. Kirilenko, A.P. Meilakhs, F.M. Shakhov, A.V. Shvidchenko, V.V. Sokolov, R.A. Babunz, A.Ya. Vul'. Diam. Relat. Mat. 75, 85 (2017).

[14] T.D. Varfolomeeva, A.G. Lyapin, S.V. Popova, N.F. Borovikov, I.P. Zibrov, V.V. Brazhkin. Inorg. Mater. 52, 4, 351 (2016).

[15] F.M. Shakhov, A.M. Abyzov, K. Takai. J. Solid State Chem. 256, 72 (2017).

[16] S. Stoll, A. Schweiger. J. Magn. Res. 178, 42 (2006).

[17] В.Ю. Осипов, Ф.М. Шахов, Н.Н. Ефимов, В.В. Минин, С.В. Кидалов, А.Я. Вуль. ФТТ 59, 6, 1125 (2017).

[18] A.I. Shames, V.Yu. Osipov, J.-P. Boudou, A.M. Panich, H.-J. von Bardeleben, F. Treussart, A.Ya. Vul'. J. Phys. D 48, 15, 155302 (2015). 